\title{
Addressing Distress in Patients With Head and Neck Cancers: A Mental Health Quality Improvement Project
}

\author{
Natalie Riblet, MD, MPH, ${ }^{\mathrm{ab}}$; Karen Skalla, MSN, APRN ${ }^{\mathrm{b}, \mathrm{c}}$; Auden McClure, MD, MPH ${ }^{\mathrm{b}, \mathrm{c}}$; \\ Karen Homa, $\mathrm{PhD}^{\mathrm{b}}$; Alison Luciano, $\mathrm{MPH}^{\mathrm{a}, \mathrm{d}}$; and Thomas H. Davis, $\mathrm{MD}^{\mathrm{b}, \mathrm{c}}$
}

\begin{abstract}
This study sought to improve mental health care for patients with head and neck cancers (HNCs) through the implementation of an evidence-based process for identifying and managing psychological distress. This process in an HNC medical oncology clinic was assessed and redesigned using quality improvement (QI) methods from November 2010 through April 2012. The redesign, starting in January 2011, involved a 2-component QI intervention: the validated NCCN Distress Thermometer and an evidence-based treatment decision algorithm. Screening processes were improved through Plan-DoStudy-Act (PDSA) cycles. Before January 2011, distress identification was based on a provider's clinical assessment. Cause-effect diagramming suggested that lack of a formalized process for distress assessment contributed to missed diagnoses. Providers were also unfamiliar with mental health resources. After implementing process changes, biweekly distress screening rates rose from $0 \%$ to $38 \%$ between January and July 2011. Furthermore, with additional PDSA cycles, these rates increased to 74\% between October 2011 and April 2012. Similar to proposed benchmarks, $84 \%(n=47)$ of newly diagnosed patients $(n=56)$ were screened. Improvement in screening was attributed to process changes and involvement of senior leadership. QI principles can be applied to the cancer setting in order to create systems of care which more reliably identify and address the needs of patients with psychological distress. (J Natl Comp Canc Netw 2014;12:1005-1013)
\end{abstract}

Symptoms of psychological distress, such as depression and anxiety, are common with any cancer but are particularly prevalent with head and neck cancers (HNCs), affecting as many as $35 \%$ of patients. ${ }^{1,2}$ Several factors likely contribute to risk, including posttreatment disfigurement and history of substance abuse. ${ }^{3-7}$ At-risk patients, however, are often not identified by providers. ${ }^{8-12}$ Left untreated, distress can contribute to poorer quality of life (QOL) and reduced survival. ${ }^{13-20}$

The importance of identifying those at risk is reflected in the NCCN Clinical Practice Guidelines in Oncology (NCCN Guidelines) for Distress Management, which recommend that cancer centers incorporate distress screening into routine care (available at NCCN.org). ${ }^{1}$ For example, NCCN recommends using the validated Distress Thermometer (NCCN-DT), a visual analogue scale that allows patients to rate their perceived level of distress in the last 7 days on a scale of 0 ("no distress") to 10 ("extreme distress"). Patients clarify the source of distress using a 39-item problem list with 5 categories: practical, family, emotional, spiritual/ religious, and physical. A score of 4 or greater suggests heightened distress and is a cue for providers to further assess identified patients and their need for treatment. ${ }^{1}$

Although distress screening is an integral part of patient-centered cancer care and is included in ASCO's Quality Oncology Practice Initiative (QOPI) measures, ${ }^{1,21-24}$ few US cancer centers have adopted formalized screening programs for distress. ${ }^{25}$ In response, a quality improvement (QI) project was developed to improve care through identifying and addressing distress in HNC medical oncology patients. Specifically, a microsystembased theory was used to apply the available evidence on distress screening to the local setting. ${ }^{26}$ This work has the potential to inform the planning and implementation of distress screening programs in cancer centers nationally. 


\section{Local Problem}

Norris Cotton Cancer Center (NCCC) is one of 41 NCI-designated Comprehensive Cancer Centers, serving patients living throughout Northern New England. Patients with HNCs are treated in the multidisciplinary $\mathrm{HNC}$ program and those requiring chemotherapy are followed up in the HNC medical oncology clinic. The clinic is staffed by a medical oncologist, a nurse practitioner, a dietician, a licensed nursing assistant (LNA), a social worker, and other ancillary staff. In 2010, the clinic cared for 548 patients with a mean age of 62.7 years $(\mathrm{SD}, 12.5)$ and $59 \%(\mathrm{n}=324)$ were men.

No institutional policy for distress screening exists, and before January 2011, the HNC medical oncology clinic lacked a formalized process, relying instead on the clinical assessment of providers to identify and manage distress. To better understand this care process, a retrospective chart review was conducted of 56 patients seen from March 2009 through January 2010. This review found that rates of identified distress, including depression, were lower than anticipated from the literature $(18 \%$ in this review vs $50 \%$ reported in the literature ${ }^{5}$ ), suggesting possible missed diagnoses. Two suicides were also noted in the last 10 years in the HNC medical oncology clinic in patients not previously identified as distressed. Patients and providers voiced concerns about lack of familiarity with available mental health resources.

\section{Intended Improvements/Aims}

In response to these findings, provision of quality, patient-centered, evidence-based mental health care for all patients in HNC medical oncology clinic became the global aim. The specific goal was to ensure that $100 \%$ of patients were screened for distress and, if indicated, received evidence-based treatment.

\section{Methods}

\section{Organizing for Improvement}

In the fall of 2010, a QI team within the HNC medical oncology clinic brought together a group of individuals with a shared interest in improving the psychosocial care of patients. The team consisted of a team leader who was a resident in Leadership Preventive Medicine (LPM) and psychiatry, a medical oncologist, an oncology nurse practitioner, and an LNA. ${ }^{27}$
The QI team received active coaching in QI principles from the team leader, who was supported by an LPM coach and participated in LPM multidisciplinary work rounds with other residents and faculty. The QI team had weekly meetings or communicated via email. Data were collected weekly and analyzed, and then distributed to HNC providers for review and discussion.

The Committee for the Protection of Human Subjects concluded that this project met criteria for quality activity without a research component and was exempt from additional review.

\section{Planning and Implementation}

Using a clinical microsystems approach, the team evaluated and worked to improve care in the $\mathrm{HNC}$ medical oncology clinic between November 2010 and April 2012 through a series of Plan-Do-StudyAct (PDSA) cycles. ${ }^{28}$ QI techniques, such as brainstorming, cause-effect analysis, and statistical process control charts, were used to connect the available evidence on distress screening to the local setting. ${ }^{26,28}$ Patient input was obtained through interviewing 28 patients and conducting a focus group of 7 patients and their caregivers. Cause-effect diagramming suggested that the lack of a formalized process for distress assessment contributed to potential missed diagnoses.

Based on these initial findings, a standardized process for distress screening and management was designed and implemented into routine care. In keeping with NCCN recommendations, the NCCNDT was used to assess for heightened psychological distress. ${ }^{1}$ A recommended cutoff score of 4 or greater was used to identify heightened distress, and emotional problems were defined as anxiety or depressive disorders. ${ }^{1}$ Process changes were initially limited to one clinic day during the week, and paper copies were used to allow flexibility in testing how routine screening can be best integrated into workflow.

Figure 1 outlines the process for distress screening and management, and administered the tool to all patients brought to the examination room, and informed patients that the purpose of the tool was to help providers better address patient needs. The provider reviewed these results during the examination and relied on an evidence-based clinical decision tree, created with input from the team, to guide management. ${ }^{1,29,30}$ Findings were documented in clinic notes and on a data collection form. Paper copies were scanned into the patient's electronic medical record (EMR). 


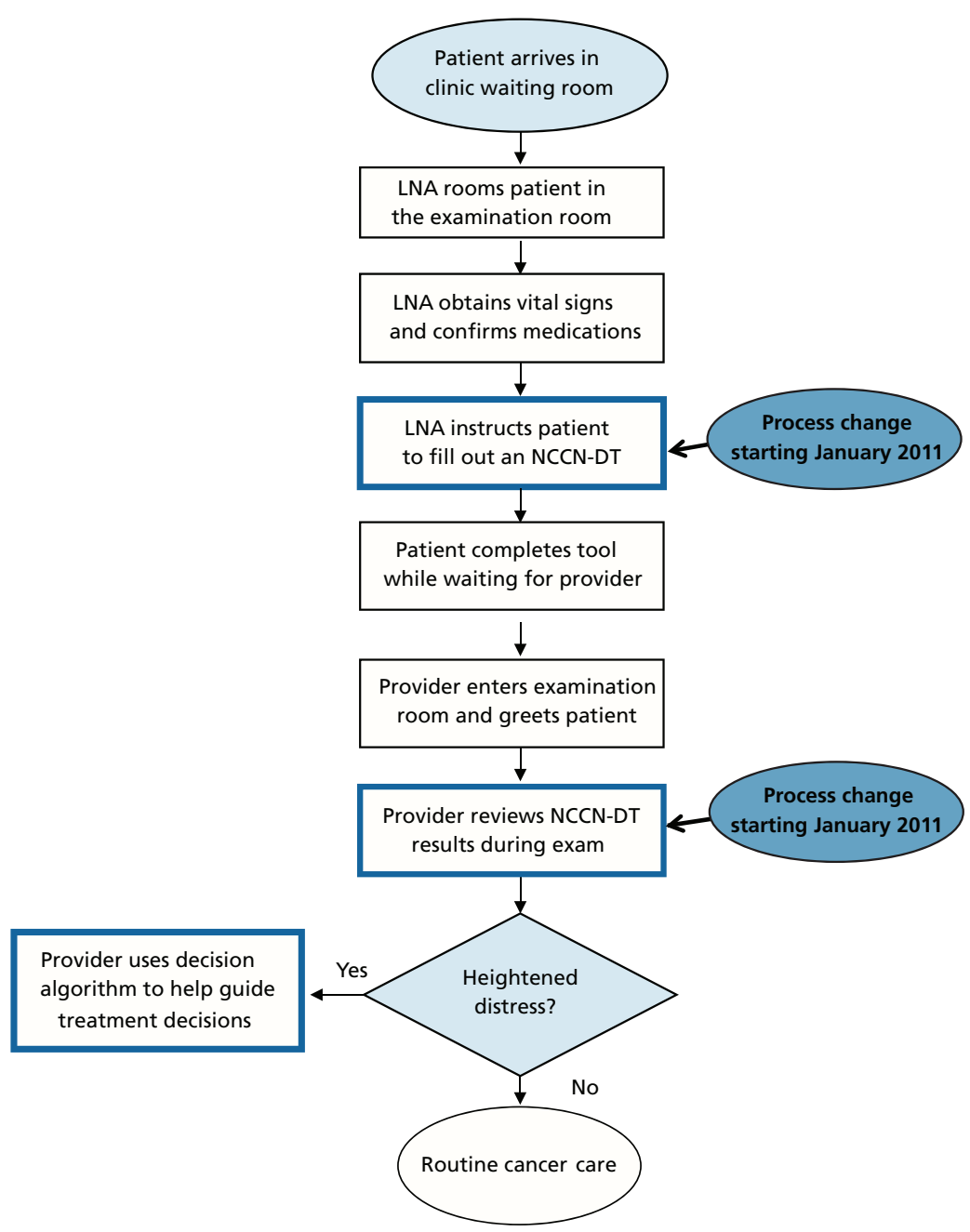

Figure 1 Process flow map for distress screening with the NCCN Distress Thermometer (NCCN-DT) in Head and Neck Cancer Medical Oncology, January 2011 through April 2012. Process revisions are outlined in dark blue.

Abbreviation: LNA, licensed nursing assistant.

\section{Outcome Measures}

The main outcome was improvement in the biweekly proportion of patients screened for distress. Secondary outcomes included proportion of patients identified with heightened distress, source of distress, and a plan for treatment of distress. In addition, patient and provider satisfaction with process changes were assessed.

\section{Analysis}

To determine whether distress screening improved over time, a proportion-chart ( $\mathrm{p}$-chart) was used. The $\mathrm{p}$-chart is a statistical process control (SPC) chart used in QI in which the proportions of patients with a given outcome are plotted over time. The SPC chart contains upper and lower control limits, which are set at 3-sigma, offering the best tradeoff between risks for type 1 and II errors. SPC charts are commonly used in QI work and differentiate between 2 types of variation: common and special cause. Processes are in statistical control when rates over time fall within the upper and lower control limits. A statistically significant change occurs when there is a special cause variation in which 1 or more points go beyond the control limits or when a process shift occurs in which 8 or more successive values fall on the same side of the overall rate. ${ }^{31}$ When a significant change occurred, the overall rate and limits were recalculated.

These results, in addition to qualitative feedback from patients and providers, guided future PDSA cycles. Specifically, special-cause signals were investigated to understand their contribution to the process (whether 
positive or negative). Once the process was in statistical control, additional small tests of changes were pursued.

Associations between categorical variables were determined using chi-square statistic.

\section{Results}

Between January 2011 and April 2012, a total of 510 patient visits occurred in the HNC medical oncology clinic. Of these, 324 (64\%) patients were successfully screened at least once for distress with the NCCNDT. A successful screen included (1) the patient was handed a hard copy of the NCCN-DT by the LNA at the beginning of the visit, (2) the provider personally reviewed the physical copy of the completed tool during the course of the visit, and (3) the tool was collected at the end of the visit and scanned into the patient's EMR by the clinic secretary. Figure 2 shows an annotated $\mathrm{p}$-chart of biweekly screening rates and describes the impact that specific PDSA cycles had on screening rates.

\section{PDSA Cycle \# 1}

Clinic notes were revised to provide visual prompts for providers to incorporate distress screening into their routine care. To address concerns that providers had insufficient knowledge, comfort skills, or resources for managing distress, the team developed an antidepressant guideline (based on current evidence) and a list of available mental health resources.

Although providers found theses resources helpful, the interventions alone had little impact on screening rates. The average screening rate for the first 4 months (January-April 2011) was 29\%. The clinic also transitioned to a new EMR in April 2011. As a result, the focus shifted as the clinic adapted to workflow changes necessitated by the change, including learning a more complex documentation and ordering system.

\section{PDSA Cycle \#2}

After implementing the new EMR, the team regrouped and brainstormed additional strategies. It became apparent that a culture shift was needed within the clinic to have distress screening become the "norm" rather than the "exception." To this end, in May 2011 a patientcentered poster was developed to educate patients about distress and encourage them to take a more active role in their care. Posters were hung in visible areas throughout the clinic. The team obtained patient-friendly pamphlets on distress from the American Cancer Society and placed these in examination rooms. ${ }^{32}$

Despite these efforts, a significant decrease in screening rates to $18 \%$ initially occurred in June

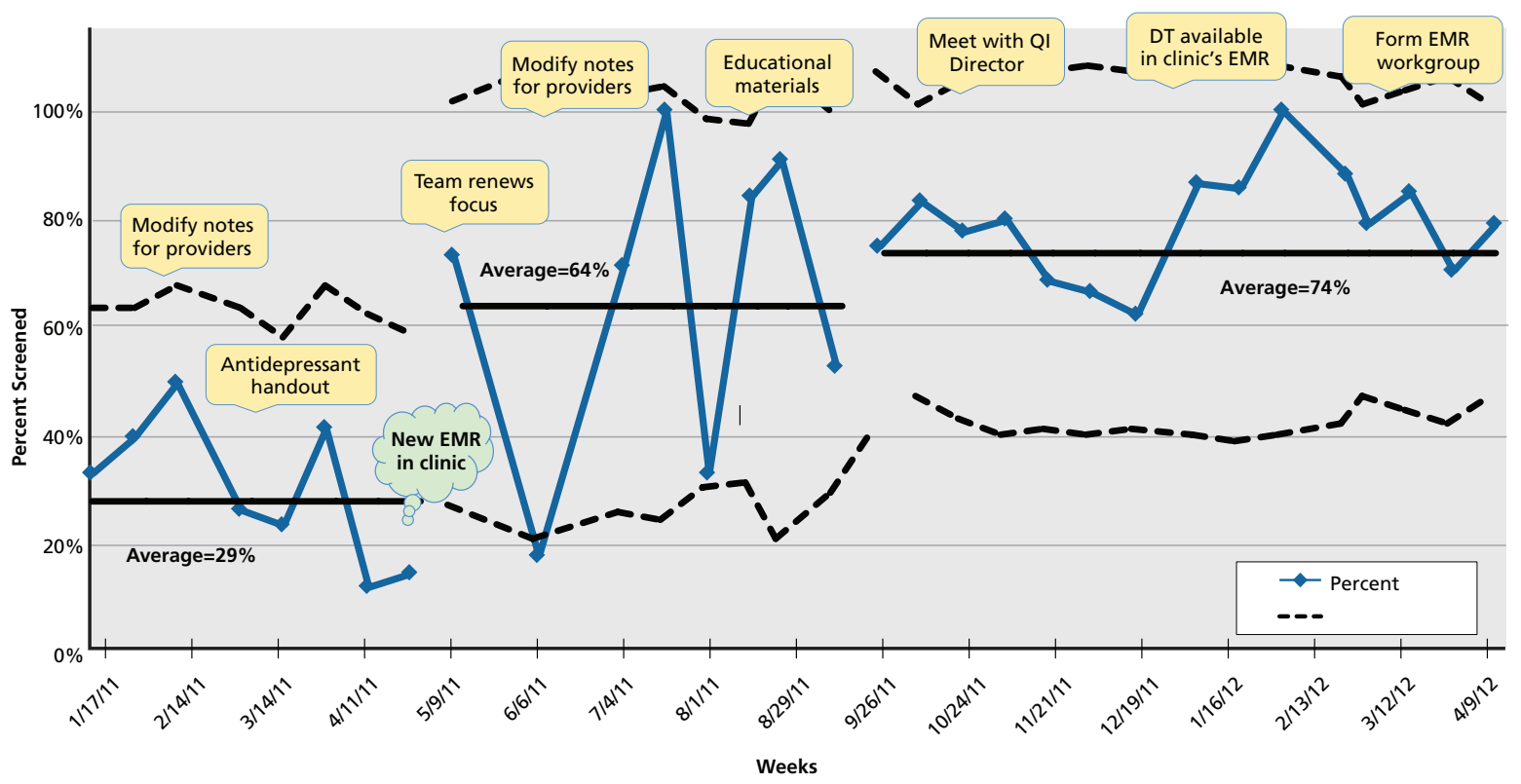

Figure 2 Proportion chart showing biweekly rates of screening with the NCCN Distress Thermometer (NCCN-DT) and associated Plan-Do-Study-Act iterations in head and neck cancer medical oncology, January 2011 through April 2012. Unplanned events are highlighted in green.

Abbreviations: EMR, electronic medical record; QI, quality improvement. 
2011. Discussions among team members suggested that several factors likely contributed. Although the LNA was now accustomed to administering the tool, she occasionally would be replaced by a floating LNA who was unfamiliar with the project and thus less familiar with (or invested in) the work. Providers and the LNA also found it difficult to adhere to the process when clinic volume was unusually high or patients had complex issues.

The team also observed that the process was not reliable or sustainable without close monitoring. Screening was highly dependent on the provider remembering to print out copies of the NCCN-DT every clinic day and hand them to the LNA. To address this process limitation, the LNA began storing paper copies in a workspace folder. Providers also made efforts to inform floating LNAs about the improvement initiative at the start of the clinic day. Finally, in response to provider feedback that the existing treatment decision algorithm was not user-friendly, it was revised and posted in the provider workroom (Figure 3).

As a result of these combined process revisions, screening rates significantly improved, with overall screening increasing to $64 \%$ between July and September 2011.

\section{PDSA Cycle \#3}

The team brainstormed additional strategies to improve screening. These included revising provider note templates to ease the burden of documentation. A significant improvement in screening occurred between early October 2011 and April 2012, with overall rates increasing to $74 \%$. These improvements were attributed partly to a shift in the clinic's culture, with screening becoming more integrated into care processes and providers gaining a deeper appreciation, through successful patient encounters, of the role that screening could play in improving patient care. This also empowered the LNA to participate and engage providers in discussions about the scores of various patients.

\section{Creating a Sustainable System}

Several ongoing barriers to screening were observed, including a heavy reliance on the LNA for the process to run smoothly. Providers also had difficulty remembering to document scores, especially in the context of a busy clinic. Furthermore, in Spring 2012, the cancer center revised its workflow patterns for patients undergoing active chemotherapy. As a result, some patients went directly to the infusion suite and bypassed the clinic altogether. Thus, providers had to remember to bring paper copies of the tool to the infusion suite or inform the nurse. Finally, the rooming process became more streamlined and patients had less time to complete the tool before providers entered the examination room.

To address these issues, the team collaborated with the cancer center's QI Director and formed a workgroup in March 2012 to consider strategies for creating a more sustainable process. The workgroup included clinic providers, the cancer center's QI Director and Support Services Director, reception, nursing, IT, social work, and volunteer services. The group identified that incorporating screening into the EMR was a critical step for project sustainability, because most of the clinic's workflow was now built around the EMR. The team explored several options, including using volunteers to assist with electronic completion of the NCCN-DTs at clinic check-in or delegating this responsibility to LNAs. Results would then be uploaded into the patient's chart and available to the provider during the examination. A pilot trial is planned as a next step.

Initial success of the project led to interest among other clinics in piloting similar distress screening protocols, representing an important step toward integrating a universal cancer center screening program.

\section{Secondary Measures}

Between January 2011 and April 2012, the clinic cared for 138 unique patients. Of these, 71.0\% $(n=98)$ were screened at least once with the NCCNDT and $33.0 \%(n=46)$ were screened several times. In addition, $83.9 \%(n=47)$ of the 56 new patients were screened, consistent with previously described benchmarks for best performing cancer centers. ${ }^{23}$

Table 1 outlines additional findings from the QI work. All patients identified with heightened distress were offered treatment, ranging from prescriptions for antidepressants to mental health referrals.

\section{Patient and Provider Responses}

Ten clinic patients were randomly selected for an interview to assess their satisfaction with process changes. These patients found that the NCCN-DT was easy and straightforward to complete. Several reported that they had been identified with heightened distress during their illness and received treat- 
Riblet et al

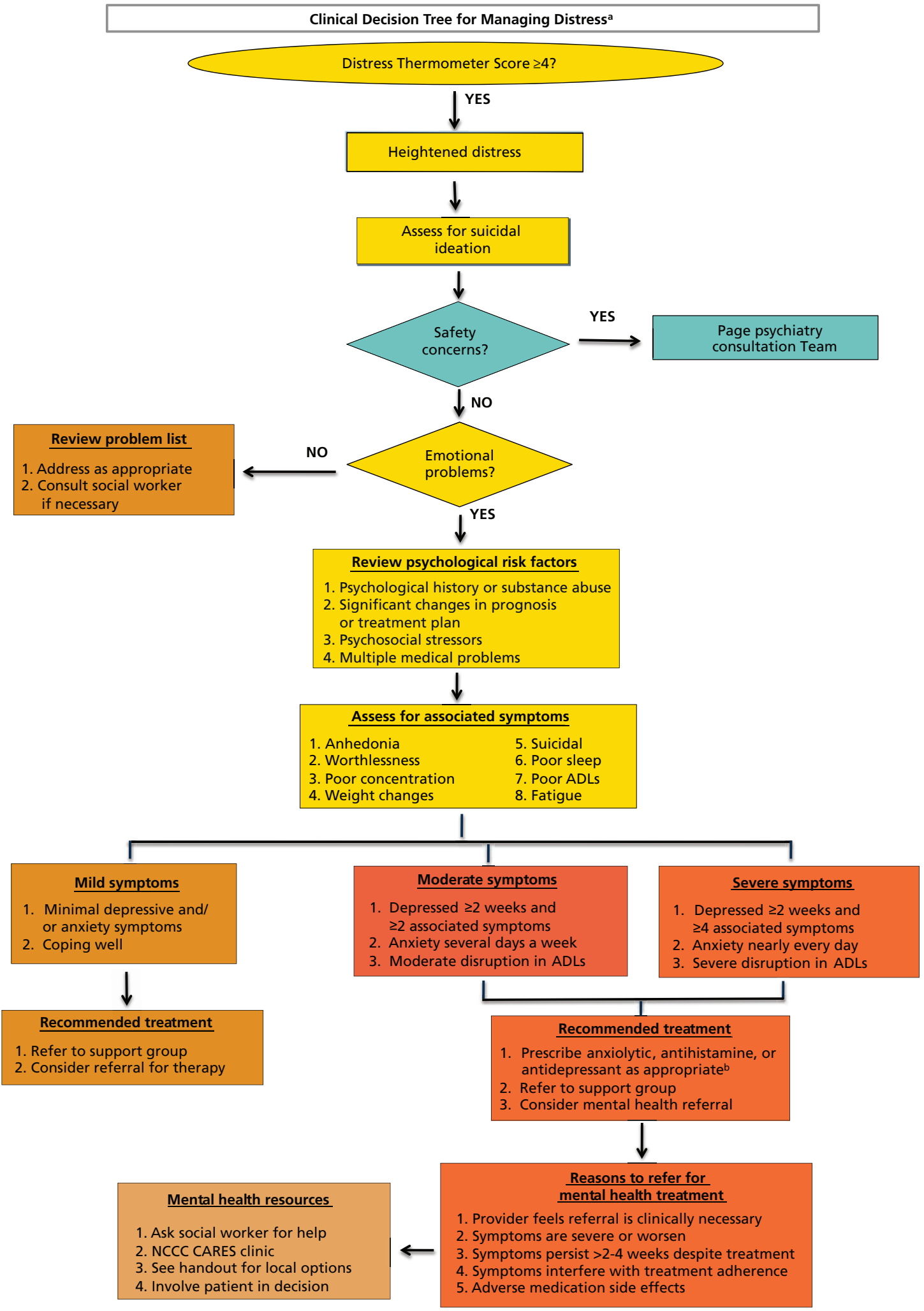

Figure 3 Treatment algorithm for managing heightened psychological distress.

Abbreviations: ADLs, activities of daily living; NCCC Cares, Norris Cotton Cancer Center Cancer and Related Events Survivorship Clinic. ${ }^{a}$ Adapted from Holland et al, ${ }^{1}$ ONS PEP Guidelines, ${ }^{29}$ and Cancer Care Ontario Symptom management tools. ${ }^{30}$

${ }^{\mathrm{b}} \mathrm{An}$ antidepressant handout was created by the team to guide treatment decisions and is available from the authors by request. 
ment that they described as beneficial. More than half of patients also expressed that integrating psychosocial care into the clinic was very important. As one patient stated, "if you're stressed and going into a situation, my feeling [is that] you won't be able to focus on what needs to happen."

Providers similarly described that the availability of distress screening data and a treatment guideline enabled them to provide more comprehensive, patient-centered care. They found it reassuring to have access to data that helped confirm clinical suspicions for heightened distress. Providers also acknowledged that some challenges exist, including incorporating distress screening in the context of a very busy clinic, or encountering patients with complex circumstances. Providers expressed that it was very important to build screening into the EMR to relieve the burden of documentation, and to help track patient needs over time.

\section{Discussion}

As a result of this QI initiative, the process for addressing the mental health needs of patients with
HNCs improved. Through a series of PDSA cycles, $84 \%$ of new patients were screened with the NCCNDT, and this screening rate met previously described benchmarks for best performing cancer centers. ${ }^{23}$ Distress screening also became an integral component of clinic work flow. In fact, although initially only $29 \%$ of patient visits were formally assessed for distress every 2 weeks, the average rose to $74 \%$ by the end of this phase of the QI work. Furthermore, providers and patients reported that the process improvement produced meaningful changes, in that providers appreciated having a tool to identify highrisk patients and an evidence-based guideline to help inform clinical care. Patients valued the opportunity to discuss this aspect of their health and to know that the treatment plan addressed their mental and physical health needs.

\section{Keys to Success}

An analysis of the factors that may contribute to diagnoses of distress being missed in the HNC medical oncology clinic highlighted several important barriers, including providers' lack of familiarity with treatment guidelines and limited access to mental health resources. To address these concerns, the screening

\begin{tabular}{|c|c|c|c|}
\hline & $\begin{array}{l}\text { Frequency } \\
\text { (n) }\end{array}$ & $\begin{array}{l}\text { Suggested } \\
\text { Benchmark }^{\mathrm{a}}\end{array}$ & Significance \\
\hline Total seen & (138) & & \\
\hline Ever screened & $71.0 \%(98)$ & & \\
\hline New patients screened ${ }^{b}$ & $83.9 \%(47)$ & $84 \%$ & \\
\hline \multicolumn{4}{|c|}{ Identified with heightened distress } \\
\hline Distress score $\geq 4$ & $47.0 \%(46)$ & & $P<.001^{c}$ \\
\hline \multicolumn{4}{|c|}{ Source of heightened distress } \\
\hline Emotional & $28.0 \%(13)$ & & \\
\hline Physical & $28.0 \%(13)$ & & \\
\hline Emotional and physical & $35.0 \%(16)$ & & \\
\hline Other ${ }^{d}$ & $9.0 \%(4)$ & & \\
\hline \multicolumn{4}{|c|}{ Treatment of heightened distress } \\
\hline Antidepressant & $41.3 \%(19)$ & & \\
\hline Mental health referral & $17.4 \%(8)$ & & \\
\hline Supportive care & $34.8 \%(16)$ & & \\
\hline Declined treatment & $4.3 \%(2)$ & & \\
\hline
\end{tabular}

${ }^{a}$ Benchmark proposed by Jacobsen et al. ${ }^{23}$

bOf 56 new patients evaluated, 47 were screened for distress.

'Compared with preintervention results, which suggested that only $18 \%$ of clinic patients (10/56) had heightened distress based on provider's clinical judgment.

dOther sources of heightened distress included family and practical problems.

eSupportive care included interventions such as referral to support groups. 
program was designed to support providers in managing psychological distress within the scope of their clinical practice. Patients with more complex needs were referred to mental health services. Providers responded favorably to these clinical decision aids.

Another integral component to the success of this QI project included the involvement of a team of individuals with a shared vision of improving the psychosocial care of patients. Providers were willing to engage in the improvement process to ensure that each patient received the best care possible at the right time. Screening rates improved significantly over time as the team worked systematically to understand barriers to care and to address them. In particular, modifying workflow patterns and engaging patients through visual aids such as posters helped foster a culture that welcomed routine distress screening. In fact, patients began to anticipate filling out an NCCN-DT as part of their clinic visit.

The decision to seek out and engage cancer center leadership was a critical component to creating momentum to sustain the screening program. The cancer center leadership recognized the important role this QI project played in furthering their mission and was invested in providing the necessary resources to make the project sustainable.

To the best of our knowledge, few studies have examined the role of QI initiatives in the context of cancer-related distress. ${ }^{33-35}$ Existing studies, however, reflect similar findings. For example, Cancer Care Ontario's QI initiative implemented the Edmonton Symptom Assessment System into multiple cancer centers and found that clinicians valued objective measurements of patient's physical and emotional status. ${ }^{34}$ These authors reported that key elements for success included involvement of senior leadership, tracking of data over time, and providing guides and algorithms to involved clinicians. ${ }^{34}$

\section{Limitations}

Factors that may make these findings less generalizable include the fact that results are representative of small sample sizes and a unique context and setting. The improvement effort was led by a team leader who had significant protected time and was supported by a dedicated coach and improvement specialist. This level of support may be difficult to replicate in other institutions.

\section{Conclusions}

QI methods can be used to design systems that more reliably identify and manage cancer-related distress. Specifically, cancer centers should consider the following steps in the design of a screening program: (1) characterize and address the unique needs of their patients and providers, (2) incorporate screening into established workflow patterns and EMR, (3) track outcomes of interest over time and share results with key stakeholders, and (4) involve senior leadership in improvement efforts.

\section{References}

1. Holland JC, Jacobsen PB, Andersen B, et al. NCCN Clinical Practice Guidelines in Oncology: Distress Management. Version 3.2012. Available at NCCN.org. Accessed May 30, 2014.

2. Zabora J, BrintzenhofeSzoc K, Curbow B, et al. The prevalence of psychological distress by cancer site. Psychooncol 2001;10:19-28.

3. Humphris GM, Rogers S, McNally D, et al. Fear of recurrence and possible cases of anxiety and depression in orofacial cancer patients. Int J Oral Maxillofac Surg 2003;32:486-491.

4. Hammerlid E, Ahlner-Elmqvist M, Bjordal K, et al. A prospective multicentre study in Sweden and Norway of mental distress and psychiatric morbidity in head and neck cancer patients. Br J Cancer 1999;80:766-774.

5. Lydiatt WM, Moran J, Burke WJ. A review of depression in the head and neck cancer patient. Clin Adv Hematol Oncol 2009;7:397-403.

6. Callahan C. Facial disfigurement and sense of self in head and neck cancer. Soc Work Health Care 2004;40:73-87.

7. El-Guebaly N. Substance abuse and mental disorders: the dual diagnosis concept. Can J Psychiatry 1990;35:261-267.

8. Newell S, Sanson-Fischer RW, Girgis A, et al. How well do medical oncologist's perceptions reflect their patient's reported physical and psychosocial problems? Data from a survey of five oncologists. Cancer 1998;83:1640-1651.

9. Sollner W, DeVries A, Steixner E, et al. How successful are oncologists in identifying patient distress, perceived social support, and need for psychosocial counseling? Br J Cancer 2001;84:179-185.

10. Fallowfield L, Ratcliffe D, Jenkins V, et al. Psychiatric morbidity and its recognition by doctors in patients with cancer. Br J Cancer 2001;84:1011-1015.

11. Kadan-Lottick NS, Vanderwerker LC, Block SD, et al. Psychiatric disorders and mental health service use in patients with advanced cancer: a report from the coping with cancer study. Cancer 2005;104:2872-2881.

12. Greenberg DB. Barriers to the treatment of depression in cancer patients. J Natl Cancer Inst Mongr 2004;32:127-135.

13. De Graeff A, de Leeuw JR, Ros WJ, et al. Pretreatment factors predicting quality of life after treatment for head and neck cancer. Head Neck 2000;22:398-407.

14. Hammerlid E, Silander E, Hornestam L, et al. Health-related quality of life three years after diagnosis of head and neck cancer—a longitudinal study. Head Neck 2001;23:113-125. 
15. Howren MB, Christensen AJ, Karnell LH, et al. Health-related quality of life in head and neck cancer survivors: impact of pretreatment depressive symptoms. Health Psychol 2010;29:65-71.

16. Lazure KE, Lydiatt WM, Denman D, et al. Association between depression and survival or disease recurrence in patients with head and neck cancer enrolled in a depression prevention trial. Head Neck 2009;31:888-892.

17. Pinquart M, Duberstein PR. Depression and cancer mortality. Psychol Med 2010;40:1797-1810.

18. Prieto JM, Blanch J, Atala J, et al. Psychiatric morbidity and impact on hospital length of stay among hematologic cancer patients receiving stem-cell transplantation. J Clin Oncol 2002;20:1907-1917.

19. Fangand CY, Schnoll RA. Impact of psychological distress on outcomes in cancer patients. Expert Rev Pharmacoecon Outcomes Res 2002;2:495-506.

20. Misono S, Weiss NS, Fann JR, et al. Incidence of suicide in persons with cancer. J Clin Oncol 2008;26:4731-4738.

21. ASCO's Quality Oncology Practice Initiative. Measures Summary, Spring 2014. Available at: http://qopi.asco.org/documents/QOPISpring-2014-Measures-Summary.pdf. Accessed June 9, 2014.

22. Schenck AP, Rokoske FS, Durham DD, et al. The PEACE project: identification of quality measures for hospice and palliative care. J Palliat Med 2010;13:1451-1459.

23. Jacobsen PB, Shibata D, Siegel EM, et al. Initial evaluation of quality indicators for psychosocial care of adults with cancer. Cancer Control 2009;16:328-334.

24. American College of Surgeons. Cancer Program Standards 2012, Version 1.2.1: Ensuring Patient-Centered. Available at http:// www.facs.org/cancer/coc/programstandards2012.html. Accessed May 30, 2014.

25. Jacobsen PB, Ransom S. Implementation of NCCN distress management guidelines by member institutions. J Natl Compr Canc Netw 2007;5:99-103.
26. Batalden PB, Davidoff F. What is "quality improvement" and how can it transform healthcare? Qual Saf Health Care 2007;16:20-23.

27. Foster T, Regan-Smith M, Murray C, et al. Residency education, preventive medicine, and population health care improvement: the Dartmouth-Hitchcock leadership preventive medicine approach. Acad Med 2008;83:390-398.

28. Nelson EC, Batalden PB, Lazar JS, et al. Improvement: foundations of knowledge for change in health care systems. In: Nelson EC, Batalden PB, Lazar JS, eds. Practice-Based Learning and Improvement: A Clinical Improvement Action Guide. 2nd edition. Oakbrook Terrace, IL: Joint Commission Resources, Inc; 2007:1-12.

29. ONS PEP Guidelines/Expert Opinion Table: Depression. Available at: www.ons.org/Research/PEP/media/ons/docs/research/outcomes/ depression/guidelines.pdf. Accessed May 30, 2014.

30. Cancer Care Ontario: symptom management tools: algorithms for managing anxiety and depression. Available at: https://www. cancercare.on.ca/toolbox/symptools/. Accessed June 23, 2014.

31. Carey RG. Improving Healthcare With Control Charts: Basic and Advanced SPC Methods and Case Studies. Milwaukee, WI: ASQ Quality Press, 2003.

32. American Cancer Society. Distress in People with Cancer. Available at: http://www.cancer.org/acs/groups/cid/documents/ webcontent/002827-pdf.pdf. Accessed May 30, 2014.

33. Adkins B, Titus-Howard T, Massey V et al. Recognizing depression in cancer outpatients: a quality improvement project. Commun Oncol 2005;2:528-533.

34. Dudgeon D, King S, Howell D, et al. Cancer Care Ontario's experience with implementation of routine physical and psychological symptom distress screening. Psychooncology 2012;21:357-364.

35. Gilbert JE, Howell D, King S, et al. Quality improvement in cancer symptom assessment and control: the provincial palliative care integration project (PPCIP). J Pain Symptom Manage 2012;43:663-678. 